\title{
Communication \\ Culture-Independent Genotyping, Virulence and Antimicrobial Resistance Gene Identification of Staphylococcus aureus from Orthopaedic Implant-Associated Infections
}

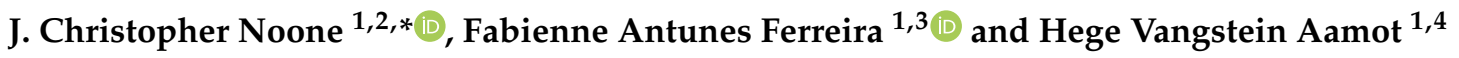 \\ 1 Department of Microbiology and Infection Control, Akershus University Hospital, 1478 Lørenskog, Norway; \\ fabienne.ferreira@ufsc.br (F.A.F.); Hege.Vangstein.Aamot@ahus.no (H.V.A.) \\ 2 Faculty of Medicine, University of Oslo, 0316 Oslo, Norway \\ 3 Departamento de Microbiologia, Imunologia e Parasitologia, Universidade Federal de Santa Catarina (UFSC), \\ Florianópolis 88040-900, Brazil \\ 4 Department of Clinical Molecular Biology (EpiGen), Akershus University Hospital and University of Oslo, \\ 1478 Lørenskog, Norway \\ * Correspondence: cnoo@ahus.no
}

Citation: Noone, J.C.; Ferreira, F.A.; Aamot, H.V. Culture-Independent Genotyping, Virulence and Antimicrobial Resistance Gene Identification of Staphylococcus aureus from Orthopaedic

Implant-Associated Infections. Microorganisms 2021, 9, 707. https:// doi.org/10.3390/microorganisms 9040707

Academic Editor: Costas

C. Papagiannitsis

Received: 10 March 2021

Accepted: 28 March 2021

Published: 30 March 2021

Publisher's Note: MDPI stays neutral with regard to jurisdictional claims in published maps and institutional affiliations.

Copyright: (c) 2021 by the authors. Licensee MDPI, Basel, Switzerland. This article is an open access article distributed under the terms and conditions of the Creative Commons Attribution (CC BY) license (https:// creativecommons.org/licenses/by/ $4.0 /)$.
Abstract: Our culture-independent nanopore shotgun metagenomic sequencing protocol on biopsies has the potential for same-day diagnostics of orthopaedic implant-associated infections (OIAI). As OIAI are frequently caused by Staphylococcus aureus, we included S. aureus genotyping and virulence gene detection to exploit the protocol to its fullest. The aim was to evaluate $S$. aureus genotyping, virulence and antimicrobial resistance genes detection using the shotgun metagenomic sequencing protocol. This proof of concept study included six patients with S. aureus-associated OIAI at Akershus University Hospital, Norway. Five tissue biopsies from each patient were divided in two: (1) conventional microbiological diagnostics and genotyping, and whole genome sequencing (WGS) of S. aureus isolates; (2) shotgun metagenomic sequencing of DNA from the biopsies. Consensus sequences were analysed using spaTyper, MLST, VirulenceFinder, and ResFinder from the Center for Genomic Epidemiology (CGE). MLST was also compared using krocus. All spa-types, one CGE and four krocus MLST results matched Sanger sequencing results. Virulence gene detection matched between WGS and shotgun metagenomic sequencing. ResFinder results corresponded to resistance phenotype. S. aureus spa-typing, and identification of virulence and antimicrobial resistance genes are possible using our shotgun metagenomics protocol. MLST requires further optimization. The protocol has potential application to other species and infection types.

Keywords: nanopore sequencing; shotgun metagenomics; culture-independent; Staphylococcus aureus; genotyping; virulence genes; antimicrobial resistance; orthopaedic implant-associated infections

\section{Introduction}

The possible ramifications of orthopaedic implant-associated infections (OIAI) are salient, including reduced function, poorer treatment outcome, and increased mortality. Delayed or poorly targeted treatment can lead to selection for antimicrobial resistant bacterial strains, revision surgery, or removal of the implant device [1,2]. The current gold standard for microbiological diagnostics of OIAI is culturing [3], which has been criticized for both its sensitivity and the time to diagnosis [4]. Culture-independent shotgun metagenomic sequencing looks to be a promising alternative in the effort to provide rapid, accurate diagnoses of OIAI.

Our recently tested protocol using nanopore shotgun metagenomic sequencing directly on tissue biopsies has the potential to facilitate same-day diagnostics and administration of targeted treatment [5]. As OIAI are most frequently caused by Staphylococcus aureus [6], we have included S. aureus protein A (spa)-typing, multilocus sequence typing (MLST), and virulence gene detection to exploit the new protocol to its fullest. 
Recent advances in the challenging area of metagenomic bacterial genome assembly in the form of the specialized long-read metagenomic assembly program metaflye [7] and sequence polishing tools such as medaka (https://github.com/nanoporetech/medaka) have made the inclusion of these analyses relatively simple. Metaflye and medaka facilitate the rapid assembly and correction of the long, error-prone nanopore $S$. aureus reads obtained from the metagenomic sequencing of OIAI in earlier work [5].

spa-typing and MLST are used in the molecular characterization of S. aureus-OIAI [8]. Additionally, the two techniques have been employed to confirm the existence of commonly occurring $S$. aureus clones in OIAI distinct to geographically restricted population groups and to confirm the concordance to the patients' own carrier clones, thus suggesting the route of infection [9].

The high frequency of $S$. aureus OIAI is, at least in part, due to the organism's array of virulence factors, some of which are used to evade the host immune response [10]. The ability to distinguish between high and low cytotoxic $S$. aureus can steer therapeutic treatment and facilitate the clearance of potentially persistent $S$. aureus infections [11].

Finally, successful treatment of an $S$. aureus OIAI is contingent upon timely and appropriate antimicrobial treatment. Conventional phenotypic antimicrobial susceptibility testing (AST) is time consuming and has been criticized for its subjectivity, lack of reproducibility, and accuracy [12-15]. Rapid and reliable prediction of antimicrobial resistance (AMR) phenotype based on detection of AMR genes can potentially reduce time to targeted OIAI treatment. However, the simple detection of AMR gene presence can be ambiguous when trying to predict AMR phenotype [5]. Improvements to the web-based ResFinder (Center for Genomic Epidemiology, CGE; https:/ / cge.cbs.dtu.dk/services/ResFinder/) have the potential to facilitate more reliable genome-based prediction AMR phenotype [16].

The aim of this work was to evaluate $S$. aureus genotyping, and virulence and antimicrobial resistance gene detection using the expanded nanopore shotgun metagenomic sequencing protocol directly from soft tissue biopsies, comparing the results to the respective current standard methods.

\section{Materials and Methods}

This prospective proof of concept study included acute OIAI patients undergoing first revision surgery at Akershus University Hospital, Norway. The patients are a sub-set of those included in an earlier study testing a culture-independent shotgun metagenomic sequencing protocol for rapid diagnostics of OIAI patients [5]. Patients with S. aureus OIAI were eligible. Five soft tissue biopsies were analysed from each patient.

Patient biopsies were taken from areas adjacent the implant. Each patient biopsy was divided into two segments. One segment was cultivated following conventional microbiological diagnostics including pathogen identification with MALDI-TOF (MatrixAssisted Laser Desorption/Ionization-Time of Flight) mass spectrometry and antimicrobial susceptibility testing in accordance with EUCAST (European Committee on Antimicrobial Susceptibility Testing) guidelines, as described in earlier work [17]. The other segment was initially frozen in $-80^{\circ} \mathrm{C}$ awaiting DNA extraction and subsequent nanopore sequencing. An analysis flowchart is depicted in Figure 1.

\subsection{DNA Extraction}

The cultured S. aureus isolate DNA was extracted using PureLink Genomic DNA Mini Kit (Invitrogen, MA, USA), following the manufacturer's protocol apart from the substitution of the lysozyme treatment by an incubation with $10 \mu \mathrm{L}$ lysostaphin $(5 \mathrm{mg} / \mathrm{mL})$ (Sigma-Aldrich, St Louis; MO, USA) in $180 \mu \mathrm{L}$ phosphate buffered saline (PBS) at $37^{\circ} \mathrm{C}$ for $45 \mathrm{~min}$.

DNA from the tissue biopsies was extracted using Ultra-Deep Microbiome Prep kit (Molzym, Bremen, Germany) optimized for enhanced degradation of human DNA (hDNA), as described in earlier work [18]. 


\section{Soft tissue OIAI biopsies}

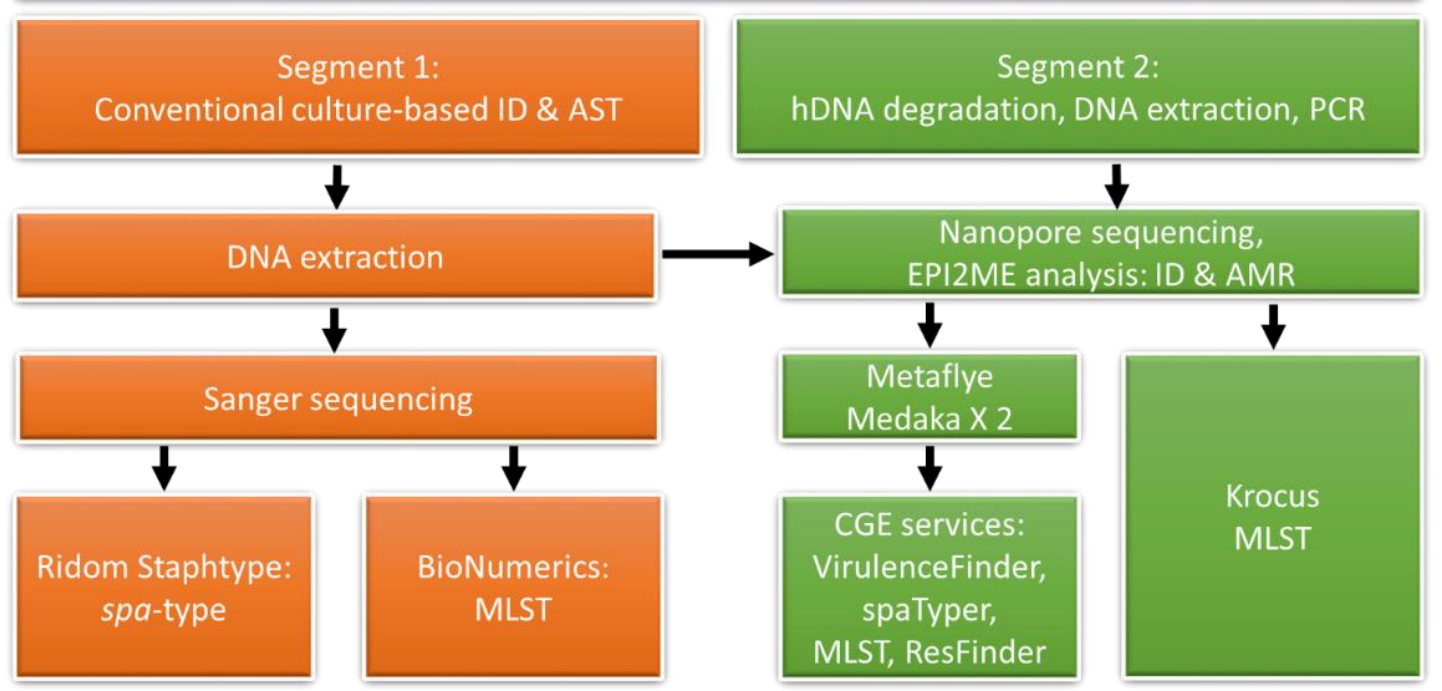

Figure 1. Flow chart of the parallel analyses of each orthopaedic implant associated infection (OIAI) patient's two biopsy segments. Following conventional analysis of $S$. aureus isolates, extracted isolate DNA was analysed comparatively to DNA extracted from the respective patients' biopsies. Additional abbreviations are as follows: AMR (antimicrobial resistance), AST (antimicrobial susceptibility testing), CGE (Center for Genomic Epidemiology), hDNA (human DNA), ID (identification), MLST (multilocus sequence typing), spa (S. aureus protein A gene).

\subsection{Spa-Typing and MLST}

Sanger-based spa-typing and MLST typing of the isolates were performed as previously described $[19,20]$. Sequencing was performed using BigDye Terminator v1.1 Cycle Sequencing Kit and the 3130xl Genetic Analyzer (Applied Biosystems, Waltham, MA, USA). Ridom Staphtype software v.2.2.1 (Ridom GmbH, Münster, Germany) was used to assign spa-type. The BioNumerics v.7.6.3 MLST online database was used for assignment of sequence type.

\subsection{Nanopore Sequencing}

WGS of the S. aureus isolates was performed using rapid barcoding kit (SQK-RBK004, Oxford Nanopore Technologies; ONT, Oxford, UK). All six isolates were sequenced on the same flow cell. Input and reagent volumes were doubled. Otherwise, library prep and sequencing were carried out following the manufacturer's protocol (RBK_9054_v2_revM_14Aug2019).

Shotgun metagenomic sequencing was performed on DNA extracted directly from patient biopsies using rapid PCR barcoding kit (SQK-RPB004) as described earlier [5].

All nanopore sequencing was performed using ONT's GridION X5 Mk1 sequencing platform and R9.4.1 FLO-MIN 106 flow cells (ONT). Each sequencing run was set to a duration of $48 \mathrm{~h}$.

\subsection{Nanopore Sequencing Data Analysis}

Raw sequencing data were analysed and basecalled using the graphical user interface MinKNOW 2.0 and Guppy basecaller v3.0.6 (ONT). ONT's cloud-based EPI2ME workflows were used for quality control and demultiplexing (Barcode), species identification (WIMP), and AMR gene identification (ARMA). EPI2ME workflows were employed using default Q-score $\geq 7$.

Both metagenomic and isolate reads were assembled with metaflye [7]. The assembled contigs were polished with two iterations of medaka (https://nanoporetech.github.io/ medaka/). The assembled metagenomic genomes were then aligned to the assembled isolate genomes in Geneious Prime (v.2021.0.3) to assess the metagenomic breadth of coverage of each's respective isolate. 
The resulting medaka consensus sequences were further analysed using spaTyper 1.0, MLST 2.0, and VirulenceFinder 2.0 services from CGE (www.genomicepidemiology.org). ResFinder 4.1 was included (both acquired antimicrobial resistance genes and chromosomal point mutations models), as previously published EPI2ME AMR gene detection using the current metagenomic protocol was suboptimal [5]. Both VirulenceFinder and ResFinder were set to $90 \%$ identity threshold and $60 \%$ minimum length threshold.

Further comparison of MLST was carried out on the uncorrected isolate and metagenomic nanopore reads using krocus, a k-mer-based typing software, designed for typing from uncorrected long-read sequence data [21].

\section{Results}

\subsection{Metaflye Assemblies}

Based on previously published metagenomic sequencing data [5], metaflye assemblies containing less than approximately 33,000 S. aureus reads yielded insufficient depth of coverage for downstream analyses (median depth $\leq 11 X$ ). Accordingly, six of 17 eligible patients' biopsies were analysed further. Their metagenomic sequencing runs ranged in $S$. aureus reads from 33,087 to $2,474,387$.

Four patients' biopsies (IDs 111, 114, 140, 141) were sequencing positive for other staphylococci: S. lugdunensis (IDs 111, 114, 140, 141), S. epidermidis (IDs 114, 140), and S. argenteus (IDs 114, 140). However, the amount of reads for these bacteria was well below $1 \%$ of the total bacterial reads, and no assemblies resulted. S. aureus was the only bacterium assembled into contigs by metaflye across the sample set. Two patients' biopsies' (IDs $139,141)$ metaflye assemblies contained several hDNA contigs in addition to S. aureus. Detailed sequencing and assembly metrics of the included patients are detailed in the supplementary data, Tables S1 and S2. The S. aureus in these six samples were identified within an hour of sequencing start using the WIMP analysis carried out in earlier work [5]. Metaflye and the two iterations of Medaka took approximately from 10 min to two hours combined, relative to the number of reads.

The metagenomic assemblies' median breadth of coverage of the isolates' S. aureus chromosome was 95\% [69.2\%-99.9\%]. Median pair-wise similarity for the metagenomicisolate chromosome alignments was 99.88\% [99.2\%-99.96\%]. Two isolates' assemblies revealed plasmids: ID 111, breadth of coverage 50\% and pair-wise similarity 99.95\%; and ID 114 , breadth of coverage $14.3 \%$ and pair-wise similarity $100 \%$ (Supplementary material, Figure S1).

\subsection{Spa-Type}

spa-typing results showed 100\% agreement between metagenomic and Sanger sequencing. The following spa-types were detected (patient ID): t2413 (ID 111), t2439 (ID 114), t122 (ID 128), t276 (ID 139), t084 (ID 140), and t5221 (ID 141). Whole genome nanopore sequencing of the respective $S$. aureus isolates matched in all cases as well.

\subsection{MLST}

The MLST results are presented in Table 1 . The metagenomic sequencing analysed with CGE resulted in one exact match with Sanger sequencing (ID 140). Raw metagenomic nanopore reads analysed with krocus matched unequivocally in four patients' samples (IDs 111, 114, 139, 141). One patient's samples (ID 140) were typed as ST6326 or ST582 due to a sequencing error in $\operatorname{arcC}$. $\operatorname{arcC}$ is one of the seven housekeeping genes sequenced to determine $S$. aureus sequence type. The sequencing errors here entailed the basecalling calling of one adenine (A) too few in a homopolymeric A region. This led to $100 \%$ matches for both arcC alleles 655 (ST6326) and 13 (ST582), leading the typing error of some reads. Krocus failed to type one patient's metagenomic sample (ID 128). 
Table 1. Overview of the S. aureus MLST results across the different protocols.

\begin{tabular}{|c|c|c|c|c|c|}
\hline Patient & $\begin{array}{c}\text { Sanger } \\
\text { BioNumerics } \\
\text { Isolate } \\
\end{array}$ & $\begin{array}{c}\text { Nanopore } \\
\text { CGE MLST } \\
\text { Isolate }\end{array}$ & $\begin{array}{c}\text { Nanopore } \\
\text { CGE MLST } \\
\text { Metagenomic }\end{array}$ & $\begin{array}{l}\text { Nanopore } \\
\text { Krocus } \\
\text { Isolate } \\
\end{array}$ & $\begin{array}{c}\text { Nanopore } \\
\text { Krocus } \\
\text { Metagenomic }\end{array}$ \\
\hline ID 111 & 15 & $\begin{array}{c}5510 ?{ }^{*} \\
\text { yqil } 99.8 \%\end{array}$ & $\begin{array}{c}5510 ? * \\
\text { yqil } 99.8 \%\end{array}$ & 15 & 15 \\
\hline ID 114 & 22 & 22 & 957 & 22 & 22 \\
\hline ID 128 & 6325 & $\begin{array}{c}6325 ? * \\
\operatorname{arcC} 99.8 \%\end{array}$ & $\begin{array}{c}6325 ? * \\
\operatorname{arcC} 99.8 \%\end{array}$ & $\begin{array}{c}6325 ? * \\
\operatorname{arcC} 99.8 \%\end{array}$ & $\begin{array}{l}\text { Not found } \\
\text { glpF } 99.9 \%\end{array}$ \\
\hline ID 139 & 30 & 4618 & 4618 & 30 & 30 \\
\hline ID 140 & 6326 & $\begin{array}{c}6326 ?{ }^{*} \\
\operatorname{arcC}, g m k, p t a, t p i \\
99.8 \%\end{array}$ & 6326 & $\begin{array}{c}6326 ?{ }^{*} \\
\operatorname{arcC}, g m k, p t a, t p i \\
99.3 \%\end{array}$ & 6326 or 582 \\
\hline ID 141 & 30 & 4618 & 4618 & $\begin{array}{c}30 * \\
\operatorname{aroE} 99.9 \%\end{array}$ & 30 \\
\hline
\end{tabular}

* Percent similarity shown where profiles were less than $100 \%$ match.

The MLST allele sequence discrepancies between nanopore sequenced samples and Sanger sequencing were most often in the form of the aforementioned missing $\mathrm{A}$ in a homopolymeric A region towards the $3^{\prime}$-end of $\operatorname{arcC}$ (Figure 2).

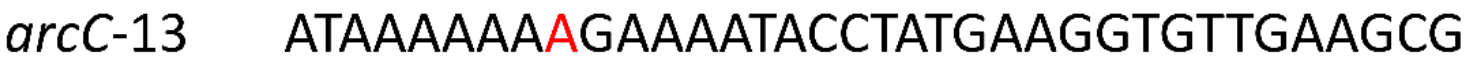

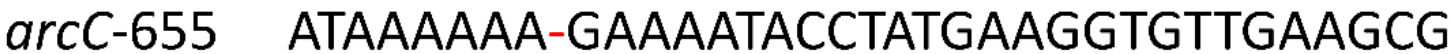

Figure 2. Example of the systematic nanopore sequencing homopolymeric A deletion. The deletion was present in all nanopore-sequenced $\operatorname{arcC}$ loci in this study, with the exception of ID 140. Shown here, is a portion of the nanopore sequenced $\operatorname{arcC}$ locus $S$. aureus isolate for patient 111 (identical to the metagenomic sequence) aligned with the Sanger sequenced isolate for the same patient. Both nanopore protocols resulted in 100\% matches for arcC allele 655 due to the basecalling error. Krocus matched Sanger sequencing, identifying this patient's arcC allele as allele 13.

Additional non-homopolymeric A deletion errors occurred in the nanopore sequenced isolate DNA from patients 111 and 140. These patients' sample sequencing results had the following sequencing errors: a cytosine $>$ thymine (C > T) substitution in yqil (ID 111); and a guanine $(\mathrm{G})$ insert in tpi, a $\mathrm{C}>\mathrm{T}$ substitution in $p t a$, and a homopolymeric $\mathrm{T}$ insertion in gmk (ID 140).

\subsection{Virulence Genes}

VirulenceFinder results of the metagenomic protocol matched the isolate WGS results $100 \%$. A complete list of all S. aureus virulence genes detected in the study is found in the supplemental data, Table S3.

\subsection{Resistance}

ResFinder AMR gene detection results of the metagenomic protocol predicted the observed culture-based AST resistance phenotypes in all cases. The phenotypes included penicillin (all samples) and tetracycline resistance (ID 128), based respectively on the detection of the genes blaZ and tetK.

The EPI2ME ARMA workflow detected the presence of genes which matched the respective AST resistance phenotypes in all cases; however, EPI2ME analysis also detected 
the presence of other putative AMR genes that were not observed in the respective AST phenotypes. The genes included tet38, sav1866, mepA, mepR, $m g r A$, arlS, arlR, and vgaA (Supplementary data, Table S4).

\section{Discussion}

This prospective proof of concept study demonstrates that, in addition to the previously reported rapid culture-free pathogen identification [5], spa-typing, identification of $S$. aureus virulence genes, and the prediction of AMR phenotype can be achieved directly from patient soft tissue biopsies using the present nanopore shotgun metagenomic sequencing protocol. However, MLST needs further optimization.

spa-typing and MLST are common genotypic screening methods for characterising $S$. aureus strains and are useful tools for detecting outbreaks and local epidemiology [8,22]. A culture-independent rapid genotyping protocol could help to quickly identify and contain local outbreaks, thus minimizing the potential consequences of such. In the present context, genotyping also serves as a quality check of sorts, assuring that the $S$. aureus isolates cultured during routine diagnostic were representative of the $S$. aureus clones of the respective patient's OIAI. spa-typing, virulence-typing, and AMR gene detection results support this. MLST, despite the basecalling issues discussed below, also seems to support this when krocus and CGE results are considered together.

spa-typing results matched $100 \%$ between methods. However, metagenomic and WGS MLST analysed using CGE were suboptimal; CGE classification matched unambiguously in one patient's samples (ID 140), uncertainly in one (ID 128), and incorrectly in the remaining four patients' samples. It should be noted that the differences in these five MLST discrepancies were sequence types that varied by a single nucleotide in four cases (IDs 114, $128,139,140$ ) and two nucleotides in the fifth (ID 114). Only a single A deletion separates ST22 and ST957 (ID 114), ST ST30 and ST4618 (IDs 139, 140), and ST6325 from a 100\% match (128). In ST15 and ST5510 (ID 111), the difference was an $\operatorname{arcC}$ A deletion and an yqil $\mathrm{C}>\mathrm{T}$ substitution. Indicating that the five metagenomic inconsistencies with Sanger sequencing were by a small margin of error.

Metagenomic krocus results matched Sanger fully in four of the six patient's samples. As shown in Table 1 however, sequence discrepancies made certain typing of MLST difficult in cases where putative basecalling errors led either to incorrect alleles being assigned (ID 140) or to imperfect allele matches (ID 128).

Nanopore sequencing is known to have some issues resolving homopolymeric regions [23]. Despite polishing the metaflye assemblies with medaka, these issues persisted. Nanopore sequencing's increased potential for erroneous basecalling in these areas at the $\operatorname{arcC}$ loci seems the cause for the present $\operatorname{arcC}$ discrepancies. ONT's newly released R10 flow cell promises improved resolution of homopolymeric regions, warranting eventual re-testing of this portion of the protocol when the R10 becomes more widely available.

The analysis of raw nanopore reads using krocus, which types and displays each uncorrected read individually in the output file, yielded more easily interpreted MLST results than the CGE analysis of the metaflye assembled and medaka polished contigs. Sequencing error was still somewhat of an issue in the krocus results of two patients' samples though (IDs 128, 140). It should be noted that krocus is dependent upon a manually updated local database. BioNumerics is updated automatically upon opening the software, and CGE synchs with pubMLST automatically.

Virulence genes detection matched $100 \%$ between nanopore shotgun metagenomic and isolate WGS. Many potential benefits of rapid identification virulence, genotype and resistance are conceivable. Identification of $S$. aureus virulence factors can give the clinician insight into an infection's pathogenesis, and, as specific anti-virulence treatments and vaccines emerge, can inform the clinician's choice of therapy [24,25]. Less cytotoxic strains of $S$. aureus, for example, have been shown to more easily evade host immunity [11]. Early identification of such strains might lead to better informed treatment decisions, helping to 
prevent chronic S. aureus infections. In addition, virulence factors such as Panton-Valentine Leukocidin (PVL) can also function as biomarkers in pathotyping strains [22].

The potential benefits of rapid AMR identification are significant. Expedient and correct OIAI antimicrobial treatment can potentially lead to reduced selection for antimicrobial resistant bacterial clones, fewer revision surgeries and device removals, increased functionality, and decreased postoperative mortality. The culture-independent metagenomic protocol's ResFinder results predicted culture-based AST resistance phenotype in all six cases. Use of this protocol could amount to a significant reduction in our current 3.5-day median time to culture-based AST results [12].

Of the samples tested here, 33,087 (ID 128) was the minimum read amount required for the protocol. Each sequencing run was set to a duration of $48 \mathrm{~h}$; however, 33,002 of the $S$. aureus reads in this run were produced within the first $24 \mathrm{~h}$ of sequencing. Sequencing hours $24-48$ produced only an additional 85 reads. The speed at which each the other five nanopore runs were able to produce $33,087 \mathrm{~S}$. aureus reads varied from about one hour to $12 \mathrm{~h}$, relative to the varying concentrations of $S$. aureus and proportions of hDNA in each sample. Nanopore sequencing allows the user to monitor the sequencing results in near real-time, and to stop the run when enough data have been accrued. The additional time required of the genotyping, virulence and resistance gene detection programs used here is negligible, adding mere minutes to the total time. Had sample ID 128 been stopped after $24 \mathrm{~h}$ of sequencing, the total time to results for the samples analysed in the present study would have ranged from about nine to $30 \mathrm{~h}$ from biopsy.

AMR gene detection of the metagenomic sequencing of these samples was carried out in earlier work using ONT's EPI2ME ARMA workflow [5]. All ARMA AMR results included genes that were not represented in the respective observed AST phenotypes. The genes included tet38, sav1866, mepA, mepR, $m g r A$, arlS, and $\operatorname{arlR}$ (Supplementary data, Table S4). These genes are reportedly present in over $99 \%$ of $S$. aureus genomes (The Comprehensive Antibiotic Resistance Database; https://card.mcmaster.ca/). They are associated with bacterial efflux systems and have the ability to confer resistance to several other antimicrobials when being expressed; however, expression is regulated by genetic variation, specific regulators, and conditions [26-29]. Their presence alone does not ensure the presence of AMR. It is, therefore, not unusual that the respective phenotypes were not reflected during AST. The presence of $v g a A$ in one patient's metagenomic data (ID 140) was due most likely to the $S$. epidermidis reads [30]. From the limited data analysed here, it would seem that the AMR gene detection results of CGE's ResFinder provides a clearer picture of the $S$. aureus AMR phenotype. In addition to AMR gene detection, ResFinder results provide a translation of genotypes into predicted phenotypes. However, as of this writing, ResFinder has only been validated for 13 bacterial species/genera of major public health relevance ( $S$. aureus included), and prediction of AMR phenotype from species other than those six may require more extensive knowledge of AMR [16].

Nanopore sequencing has been successfully employed by others for outbreak analysis [31], WGS spa-typing and virulence typing [32] and targeted MLST [33] of S. aureus. These analyses were performed on isolates, however.

The study's limitations lie primarily in the small sample group that contained "sufficient" $S$. aureus sequencing reads. Additionally, as this is a single centre study in a geographic location with a low prevalence of AMR, the antimicrobial resistance detection results might translate differently to areas with a greater pathogen diversity and prevalence of AMR. Norway is a country with a relatively low prevalence of methicillin resistant $S$. aureus (MRSA). No MRSA were among the samples included in the present study. In the current report, the Norwegian program for the surveillance of antimicrobial resistance (NORM) reports that MRSA comprised no more than $1.3 \%$ of the S. aureus isolated tested from human sources [34]. More diverse AMR among the $S$. aureus strains might have given better insight into the protocol's ability to predict AMR phenotype.

The metagenomic read threshold for the feasibility of the present protocol apparently lies roughly between 10,000 and 33,000 S. aureus reads and a median depth of coverage 
somewhere between 11 and 33. When aligned to the isolate assemblies, the metagenomic assemblies' median breadth of coverage of the isolates' S. aureus chromosome was 95\% [69.2\%-99.9\%], and pair-wise identity matched a median of $99.88 \%$. In two patients samples' isolates' assemblies, plasmids were observed. Plasmid coverages were $50 \%$ and $14.3 \%$. Assembly breadth of coverage was not proportional to amount of metagenomic nanopore reads, but rather an apparent partiality in the PCR amplification of certain $S$. aureus genomic fragments during pre-sequencing sample preparation. This is somewhat troubling in that it implies that the genes necessary for spa-typing, MLST, and virulence and AMR gene detection might not have been present for downstream analyses had they been carried on these missing areas. This was not an issue in the current work.

\section{Conclusions}

These results indicate that $S$. aureus spa-typing, and identification of virulence and antimicrobial resistance genes are possible using nanopore shotgun metagenomics sequencing directly on tissue biopsies analysed with the protocol presented here. Culture-free detection of virulence genes and prediction of AMR phenotype can contribute to more timely and more accurately targeted treatment of OIAI. The protocol requires optimization before it can be reliably used for MLST classification of S. aureus. Apart from spa-typing, specific to $S$. aureus, the protocol has the potential to be used in the analysis of other species and types of infections.

Supplementary Materials: The following are available online at https:/ /www.mdpi.com/article/10 $.3390 /$ microorganisms $9040707 /$ s1. Figure S1. Alignment of the assembled metagenomic assemblies with each's respective isolate. Table S1. Nanopore sequencing QC metrics. Table S2. metaflye assembly metrics. Table S3. VirulenceFinder results. Table S4. Results of resistance phenotype (AST) and resistance genes detected.

Author Contributions: Conceptualization, H.V.A.; methodology, J.C.N., F.A.F., H.V.A.; software, J.C.N., F.A.F. and H.V.A.; validation, J.C.N., F.A.F. and H.V.A.; formal analysis, J.C.N., F.A.F. and H.V.A.; investigation, J.C.N., F.A.F. and H.V.A.; resources, H.V.A.; data curation, J.C.N.; writingoriginal draft preparation, J.C.N.; writing-review and editing, J.C.N., F.A.F. and H.V.A.; visualization, J.C.N.; supervision, H.V.A.; project administration, H.V.A.; funding acquisition, H.V.A. All authors have read and agreed to the published version of the manuscript.

Funding: Funding for this work was provided through Akershus University Hospital internal strategic research funds (\#267923) and South-Eastern Norway Regional Health Authority (\#278903 and \#298904).

Institutional Review Board Statement: The study has been performed in accordance with the Declaration of Helsinki. The Regional Committees for Medical and Health Research Ethics (SouthEast, 2016/1929) and the Akershus University Hospital's local Data Protection Officer (17/024) approved this study.

Informed Consent Statement: Informed consent was obtained from all subjects involved in the study.

Data Availability Statement: The S. aureus sequencing data have been deposited in the National Center for Biotechnology Information (NCBI) under bioproject ID PRJNA714681. Additional data provided upon request. Requests can be directed to the corresponding author.

Conflicts of Interest: The authors declare no conflict of interest.

\section{References}

1. Karachalios, T.; Koutalos, A.; Komnos, G. Management Strategies for Infected Total Hip Arthroplasty. A Critical Appreciation of Problems and Techniques. HIP Int. 2014, 24, S44-S47. [CrossRef]

2. Siljander, M.P.; Sobh, A.H.; Baker, K.C.; Baker, E.A.; Kaplan, L.M. Multidrug-Resistant Organisms in the Setting of Periprosthetic Joint Infection-Diagnosis, Prevention, and Treatment. J. Arthroplast. 2018, 33, 185-194. [CrossRef]

3. Parvizi, J.; Zmistowski, B.; Berbari, E.F.; Bauer, T.W.; Springer, B.D.; Della Valle, C.J.; Garvin, K.L.; Mont, M.A.; Wongworawat, M.D.; Zalavras, C.G. New Definition for Periprosthetic Joint Infection: From the Workgroup of the Musculoskeletal Infection Society. Clin. Orthop. Relat. Res. 2011, 469, 2992-2994. [CrossRef] 
4. $\quad$ Eckburg, P.B.; Bik, E.M.; Bernstein, C.N.; Purdom, E.; Dethlefsen, L.; Sargent, M.; Gill, S.R.; Nelson, K.E.; Relman, D.A. Diversity of the Human Intestinal Microbial Flora. Science 2005, 308, 1635-1638. [CrossRef]

5. Noone, J.C.; Helmersen, K.; Leegaard, T.M.; Skråmm, I.; Aamot, H.V. Rapid Diagnostics of Orthopaedic Implant-Associated Infections Using Nanopore Shotgun Metagenomic Sequencing on Tissue Biopsies. Microorganisms 2021, 9, 97. [CrossRef] [PubMed]

6. Arciola, C.R.; Campoccia, D.; Montanaro, L. Implant Infections: Adhesion, Biofilm Formation and Immune Evasion. Nat. Rev. Microbiol. 2018, 16, 397-409. [CrossRef] [PubMed]

7. Kolmogorov, M.; Bickhart, D.M.; Behsaz, B.; Gurevich, A.; Rayko, M.; Shin, S.B.; Kuhn, K.; Yuan, J.; Polevikov, E.; Smith, T.P.L.; et al. MetaFlye: Scalable Long-Read Metagenome Assembly Using Repeat Graphs. Nat. Methods 2020, 17, 1103-1110. [CrossRef]

8. Montanaro, L.; Ravaioli, S.; Ruppitsch, W.; Campoccia, D.; Pietrocola, G.; Visai, L.; Speziale, P.; Allerberger, F.; Arciola, C.R. Molecular Characterization of a Prevalent Ribocluster of Methicillin-Sensitive Staphylococcus Aureus from Orthopedic Implant Infections. Correspondence with MLST CC30. Front. Cell. Infect. Microbiol. 2016, 6. [CrossRef]

9. Skramm, I.; Moen, A.E.F.; Asbjorn, A.; Bukholm, G. Surgical Site Infections in Orthopaedic Surgery. J. Bone Jt. Surg. Am. 2014, 96, 882-888.

10. Thammavongsa, V.; Kim, H.K.; Missiakas, D.; Schneewind, O. Staphylococcal Manipulation of Host Immune Responses. Nat. Rev. Microbiol. 2015, 13, 529-543. [CrossRef] [PubMed]

11. Tuchscherr, L.; Pöllath, C.; Siegmund, A.; Deinhardt-Emmer, S.; Hoerr, V.; Svensson, C.M.; Figge, M.T.; Monecke, S.; Löffler, B. Clinical S. Aureus Isolates Vary in Their Virulence to Promote Adaptation to the Host. Toxins 2019, 11, 135. [CrossRef] [PubMed]

12. Aamot, H.V.; Noone, J.C.; Skråmm, I.; Truls, M. Are Conventional Microbiological Diagnostics Sufficiently Expedient in the Era of Rapid Diagnostics? Evaluation of Conventional Microbiological Diagnostics of Orthopedic Implant- Associated Infections (OIAI) Are Conventional Microbiological Diagnostics. Acta Orthop. 2020. [CrossRef]

13. Pedersen, S.K.; Wagenaar, J.A.; Vigre, H.; Roer, L.; Mikoleit, M.; Aidara-Kane, A.; Cawthorne, A.L.; Aarestrup, F.M.; Hendriksen, R.S. Proficiency of WHO Global Foodborne Infections Network External Quality Assurance System Participants in the Identification and Susceptibility Testing of Thermo-Tolerant Campylobacter Spp. from 2003-2012. bioRxiv 2018, 56, 1-9. [CrossRef]

14. Hendriksen, R.S.; Seyfarth, A.M.; Jensen, A.B.; Whichard, J.; Karlsmose, S.; Joyce, K.; Mikoleit, M.; Delong, S.M.; Weill, F.X.; AidaraKane, A.; et al. Results of Use of Who Global Salm-Surv External Quality Assurance System for Antimicrobial Susceptibility Testing of Salmonella Isolates from 2000 to 2007. J. Clin. Microbiol. 2009, 47, 79-85. [CrossRef]

15. Diekema, D.J.; Lee, K.; Raney, P.; Herwaldt, L.A.; Doern, G.V.; Tenover, F.C. Accuracy and Appropriateness of Antimicrobial Susceptibility Test Reporting for Bacteria Isolated from Blood Cultures. J. Clin. Microbiol. 2004, 42, 2258-2260. [CrossRef] [PubMed]

16. Bortolaia, V.; Kaas, R.S.; Ruppe, E.; Roberts, M.C.; Schwarz, S.; Cattoir, V.; Philippon, A.; Allesoe, R.L.; Rebelo, A.R.; Florensa, A.F.; et al. ResFinder 4.0 for Predictions of Phenotypes from Genotypes. J. Antimicrob. Chemother. 2020, 75, 3491-3500. [CrossRef]

17. Aamot, H.V.; Johnsen, B.O.; Skråmm, I. Rapid Diagnostics of Orthopedic Implant-Associated Infections Using Unyvero ITI Implant and Tissue Infection Application Is Not Optimal for Staphylococcus Species Identification. BMC Res. Notes 2019, 12, 725. [CrossRef] [PubMed]

18. Helmersen, K.; Aamot, H.V. DNA Extraction of Microbial DNA Directly from Infected Tissue: An Optimized Protocol for Use in Nanopore Sequencing. Sci. Rep. 2020, 10, 2985. [CrossRef] [PubMed]

19. Blomfeldt, A.; Jørgensen, S.B.; Helmersen, K.; Eskonsipo, P.K.J.; Aamot, H.V. Is Increased Staphylococcus Aureus Colonisation during Military Service Caused by Specific Genotypes? Molecular Examination of Long-Term Carriage in a Prospective Cohort Study. APMIS 2020, 7, 170-177. [CrossRef]

20. Enright, M.C.; Day, N.P.J.; Davies, C.E.; Peacock, S.J.; Spratt, B.G. Multilocus Sequence Typing for Characterization of MethicillinResistant and Methicillin-Susceptible Clones of Staphylococcus Aureus. J. Clin. Microbiol. 2000, 38, 1008-1015. [CrossRef]

21. Page, A.J.; Keane, J.A. Rapid Multi-Locus Sequence Typing Direct from Uncorrected Long Reads Using Krocus. PeerJ 2018, 2018, e5233. [CrossRef] [PubMed]

22. Stefani, S.; Chung, D.R.; Lindsay, J.A.; Friedrich, A.W.; Kearns, A.M.; Westh, H.; MacKenzie, F.M. Meticillin-Resistant Staphylococcus Aureus (MRSA): Global Epidemiology and Harmonisation of Typing Methods. Int. J. Antimicrob. Agents 2012, 39, $273-282$. [CrossRef] [PubMed]

23. Kono, N.; Arakawa, K. Nanopore Sequencing: Review of Potential Applications in Functional Genomics. Dev. Growth Differ. 2019, 61, 316-326. [CrossRef] [PubMed]

24. Kurlenda, J.; Grinholc, M. Alternative Therapies in Staphylococcus Aureus Diseases. Acta Biochim. Pol. 2012, 59, 171-184. [CrossRef]

25. Kane, T.L.; Carothers, K.E.; Lee, S.W. Virulence Factor Targeting of the Bacterial Pathogen Staphylococcus Aureus for Vaccine and Therapeutics. Curr Drug Targets 2018, 19, 111-127. [CrossRef]

26. Truong-Bolduc, Q.C.; Dunman, P.M.; Strahilevitz, J.; Projan, S.J.; Hooper, D.C. MgrA Is a Multiple Regulator of Two New Efflux Pumps in Staphylococcus Aureus. J. Bacteriol. 2005, 187, 2395-2405. [CrossRef] 
27. McAleese, F.; Petersen, P.; Ruzin, A.; Dunman, P.M.; Murphy, E.; Projan, S.J.; Bradford, P.A. A Novel MATE Family Efflux Pump Contributes to the Reduced Susceptibility of Laboratory-Derived Staphylococcus Aureus Mutants to Tigecycline. Antimicrob. Agents Chemother. 2005, 49, 1865-1871. [CrossRef] [PubMed]

28. Dawson, R.J.P.; Locher, K.P. Structure of the Multidrug ABC Transporter Sav1866 from Staphylococcus Aureus in Complex with AMP-PNP. FEBS Lett. 2007, 581, 935-938. [CrossRef]

29. Costa, S.S.; Viveiros, M.; Amaral, L.; Couto, I. Multidrug Efflux Pumps in Staphylococcus Aureus: An Update. Open Microbiol. J. 2013, 7, 59-71. [CrossRef]

30. Tessé, S.; Trueba, F.; Berthet, N.; Hot, C.; Chesneau, O. Resistance Genes Underlying the LSA Phenotype of Staphylococcal Isolates from France. Antimicrob. Agents Chemother. 2013, 57, 4543-4546. [CrossRef] [PubMed]

31. Ferreira, F.A.; Helmersen, K.; Visnovska, T.; Jørgensen, S.B.; Aamot, H.V. Rapid Nanopore-Based DNA Sequencing Protocol of Antibiotic-Resistant Bacteria for Use in Surveillance and Outbreak Investigation. Microb. Genom. 2020. Accepted, pending publication.

32. Durand, G.; Javerliat, F.; Bes, M.; Veyrieras, J.B.; Guigon, G.; Mugnier, N.; Schicklin, S.; Kaneko, G.; Santiago-Allexant, E.; Bouchiat, C.; et al. Routine Whole-Genome Sequencing for Outbreak Investigations of Staphylococcus Aureus in a National Reference Center. Front. Microbiol. 2018, 9, 1-12. [CrossRef] [PubMed]

33. Liou, C.H.; Wu, H.C.; Liao, Y.C.; Lauderdale, T.L.Y.; Huang, I.W.; Chen, F.J. Nanomlst: Accurate Multilocus Sequence Typing Using Oxford Nanopore Technologies Minion with a Dual-Barcode Approach to Multiplex Large Numbers of Samples. Microb. Genom. 2020, 6, 1-8. [CrossRef] [PubMed]

34. NORM NORM-VET. 2019. Available online: https:/ /www.vetinst.no/en/surveillance-programmes/norm-norm-vet-report (accessed on 27 March 2021). 